\title{
A systematic literature review of community pharmacist-led interventions to optimize the use of antibiotics
}

\author{
Maarten Lambert ${ }^{1}$, Chloé Smit ${ }^{2}$, Stijn De Vos ${ }^{1}$, Ria Benko $^{3}$, Carles Llor $^{4}$, John Paget ${ }^{5}$, \\ Kathryn Briant ${ }^{6}$, Lisa Pont ${ }^{2}$, Liset van Dijk ${ }^{7}$, and Katja Taxis ${ }^{1}$ \\ ${ }^{1}$ University of Groningen \\ ${ }^{2}$ University of Technology Sydney \\ ${ }^{3}$ University of Szeged Albert Szent-Gyorgyi Health Centre \\ ${ }^{4}$ University institute in primary care research Jordi Gol \\ ${ }^{5}$ NIVEL \\ ${ }^{6}$ Health Care Consumers' Association \\ ${ }^{7}$ Nederlands Instituut voor Onderzoek van de Gezondheidszorg
}

June 24, 2021

\begin{abstract}
Objectives. The aim of this systematic review is to assess the effects of community pharmacist-led interventions to optimize the use of antibiotics and identify which interventions are most effective. Methods. This review was conducted according to the PRISMA-P guidelines (PROSPERO: CRD42020188552). PubMed, EMBASE and the Cochrane Central Register of Controlled Trials were searched for (randomised) controlled trials. Included interventions were required to target antibiotic use, be set in the community pharmacy context and be pharmacist-led. Primary outcomes were quality of antibiotic supply and adverse effects while secondary outcomes included patient reported outcomes. Risk of bias was assessed using the 'Cochrane suggested risk of bias criteria' and narrative synthesis of primary outcomes conducted. Results. Seventeen studies were included covering in total 3,822 patients (mean age 45.6 years, $61.9 \%$ female). Most studies used educational interventions. Three studies reported on primary outcomes, twelve on secondary outcomes and two on both. Three studies reported improvements in quality of dispensing where interventions led to more intensive symptom assessment and a reduction of OTC or wrong choice antibiotic supply. Some interventions led to higher consumer satisfaction, effects on adherence were mixed. All studies had unclear or high risks of bias across at least one domain, with large heterogeneity between studies. Conclusions. Our review suggests some possible positive results from pharmacist-led interventions, but the role of the pharmacist needs to be expanded. This review should be interpreted as exploratory research, as more high-quality research is needed. Authors did not receive funding for the review.
\end{abstract}

\section{Hosted file}

BJCP_Full manuscript systematic review pharmacy interventions antibiotic resistance_Maarten Lambert.doc available at https://authorea.com/users/421696/articles/527619-a-systematic-literaturereview-of-community-pharmacist-led-interventions-to-optimize-the-use-of-antibiotics 